\title{
Organic light-emitting diodes with $30 \%$ external quantum efficiency based on a horizontally oriented emitter
}

By Sei-Yong Kim, Won-Ik Jeong, Christian Mayr, Young-Seo Park, Kwon-Hyeon Kim, JeongHwan Lee, Chang-Ki Moon, Wolfgang Brütting, and Jang-Joo Kim*

\section{((Optional Dedication))}

[*] Prof. Jang-Joo Kim, Sei-Yong Kim, Dr. Won-Ik Jeong, Dr. Young-Seo Park, KwonHyeon Kim, Jeong-Hwan Lee, and Chang-Ki Moon

Department of Materials Science and Engineering and Center for Organic Light Emitting Diode

Seoul National University, Seoul 151-742, South Korea

E-mail: jikim@snu.ac.kr

Christian Mayr, and Prof. Wolfgang Brütting

Institute of Physics

University of Augsburg, 86135 Augsburg, Germany

Keywords: Organic light-emitting diodes, Phosphorescent emitter, Emitter orientation, Optical simulation, Exciplex forming host, Ultra-high efficiency

High efficiency phosphorescent organic light emitting diodes (OLEDs) doped with $\operatorname{Ir}(\mathrm{ppy})_{2}(\mathrm{acac})[\mathrm{bis}(2-$ phenylpyridine)iridium(III)-acetylacetonate] in an exciplex forming cohost have been optically analyzed. This emitter has a preferred orientation with the horizontal to vertical dipole ratio of $0.77: 0.23$ as compared to $0.67: 0.33$ in the isotropic case. Theoretical analysis based on the orientation factor $(\Theta$, the ratio of the horizontal dipoles to total dipoles) and the photoluminescence quantum yield $\left(q_{P L}\right)$ of the emitter predicts that the maximum external quantum efficiency (EQE) of the OLEDs with this emitter is about $30 \%$ which matches very well with the experimental data, indicating that the electrical loss of the OLEDs is negligible and the device structure can be utilized as a platform to demonstrate the validity of optical modeling. Based on the results, we claim that the maximum EQE achievable with a certain emitting dye in a host can be predicted by just measuring $q_{P L}$ and $\Theta$ in a neat film on 
glass without fabricating devices and offer a universal plot of the maximum EQE as functions of $q_{P L}$ and $\Theta$.

\section{Introduction}

Organic light emitting diodes (OLEDs) with horizontally aligned emitters have the potential to achieve high external quantum efficiency $(\mathrm{EQE})$ without outcoupling enhancement layers. An emitter with a horizontal transition dipole moment results in much higher outcoupling efficiency than the vertically aligned dipole as demonstrated in polymers and vacuum evaporated organic molecules. ${ }^{[1-8]}$ Recently, not only fluorescent molecules but also some phosphorescent dyes are reported to have preferred horizontal dipoles where high EQE over $30 \%$ is expected. ${ }^{[9-13]}$ Unfortunately no experimental data have yet demonstrated the potential of horizontally oriented phosphorescent dyes to get high efficiencies over $30 \%$ to our best knowledge because of the lack of a device structure to get perfect electron and hole balance. Moreover, the most commonly used green phosphorescent dye, $\operatorname{Ir}(\mathrm{ppy})_{3}$, doped in CBP was reported to have isotropic distribution of emitting dipoles. ${ }^{[13]}$ Thus, it is important to develop or find a host/guest system that has preferentially horizontal dipoles as well as a device structure with nearly $100 \%$ charge balance, i.e. without electrical loss, to demonstrate the validity of the prediction of the optical model.

Recently our group reported a very high efficiency green phosphorescent OLED with the EQE of $29.1 \%$ and extremely low roll-off of efficiency at high current density. ${ }^{[14]}$ In this device $\operatorname{Ir}(\mathrm{ppy})_{2}(\mathrm{acac})$ [bis(2-phenylpyridine)iridium(III)-acetylacetonate] was doped in the exciplex forming co-host system of TCTA [4, 4', 4 "-Tri (N-carbazolyl) triphenylamine] and B3PYMPM [bis-4,6-(3,5-di-3-pyridylphenyl)-2-methylpyrimi-dine]. The high efficiency of the OLED with low roll-off of efficiency indicates that the electrical loss (including charge balance and exciton-polaron quenching) seems to be negligible in the device so that it can be used as a platform to analyze the outcoupling efficiency and the factors influencing the EQE 
and eventually validate the prediction of an optical model.

In this paper, we demonstrate by optical analysis that the phosphorescent emitter $\operatorname{Ir}(\mathrm{ppy})_{2}(\mathrm{acac})$ in the device has a preferred non-isotropic orientation with horizontal to vertical dipole ratio of 0.77:0.23 $(\Theta=0.77)$, and the device has the maximum EQE of $30 \%$ based on the photoluminescence (PL) quantum yield $\left(q_{P L}\right)$ of 0.94 in the thin film and 100\% charge balance. The theoretical prediction agrees very well with the experimental data, validating the optical model used for the prediction of the EQE. Based on the validation, we offer a universal plot of maximum efficiency achievable with different values of $q_{P L}$ and $\Theta$ in a dye doped EML without fabricating devices. The optical analysis indicates that OLEDs with EQE higher than $40 \%$ can be realized without any extra light extraction layers, if phosphorescent dyes with $q_{P L}$ and $\Theta$ over $95 \%$ are used.

\section{Simulation of external quantum efficiency}

The EQE of an OLED has been expressed by the following equation: ${ }^{[15]}$

$$
\eta_{E Q E}=\gamma \times \eta_{S / T} \times q_{P L} \times \eta_{\text {out }}
$$

where $\gamma$ is the charge balance factor, $\eta_{S / T}$ is the singlet-triplet factor $\left(\eta_{S / T}=0.25\right.$ for fluorescent, $\eta_{S / T}=1$ for phosphorescent emitter), $q_{P L}$ is the PL quantum yield, and $\eta_{\text {out }}$ is the outcoupling efficiency of the emitted light. However, the quantum efficiency of an emitter in a micro-cavity structure is influenced by the orientation of the emitter, the local electric field at the dipole position and the proximity to a metal layer. The $\eta_{\text {out }}$ is influenced not only by the device structure but also by the orientation of emitting dipoles so that the EQE must be modified as follows: $:^{[13,16]}$

$$
\eta_{E Q E}=\gamma \times \eta_{S / T} \times q_{e f f}\left(q_{P L}, \Theta, \Gamma\right) \times \eta_{\text {out }}(\Theta, \Gamma)
$$

where $q_{\text {eff }}$ is the effective quantum yield that describes the probability of radiative exciton 
decay in an optical cavity structure (i.e. the Purcell effect ${ }^{[17]}$, which is generally depending on $q_{P L}, \Theta$, and the geometric factor of the device $(\Gamma)$ including the device structure and the location of the emission zone in the device. In a similar manner, $\eta_{\text {out }}$ is influenced by $\Theta$ and $\Gamma$. The $\gamma$ value is often assumed to be unity in state-of-the-art OLEDs, however, this value is not a constant but a fitting parameter in this study. With separately measured values of $q_{P L}$ and $\Theta$, and using known information of the device structure, we can now calculate $q_{\text {eff }}$ and $\eta_{\text {out }}$ from a classical dipole model and then fit to the experimentally obtained EQE to extract $\gamma$. Therefore, we can exactly analyze the effect of the emitter orientation, the electrical loss as well as the device structure on EQE by fitting theoretically predicted EQE to experimental data. Details of the method for calculating $\eta_{\text {out }}$ were described elsewhere. ${ }^{[18-21]}$ We assumed that the emission zone is located in the middle of the EML in OLEDs, which is a reasonable assumption for the device having a uniformly distributed profile of the emission zone such as the co-host system used here. ${ }^{[22]}$

A schematic diagram of the device structure and the chemical structure of the materials used in this study are shown in Fig. 1a. The refractive indices of the organic layers, except for B3PYMPM, were measured by a spectroscopic ellipsometer. ${ }^{[23]}$ The refractive indices of B3PYMPM, glass substrate, ITO and metal are taken from literature. ${ }^{[7,24,25]}$ The refractive indices of the undoped co-host layer (TCTA:B3PYMPM, 1:1 molar ratio) measured by a spectroscopic ellipsometer are displayed in Fig. 1b. We ignored the modification of the optical constant by the doping of the phosphorescent dye molecules due to the low doping concentration $(<10 \%)$. The optical constants of the co-host system show strong anisotropy with an ordinary refractive index $\left(n_{o}\right)$ of 1.8342 and an extra-ordinary refractive index $\left(n_{e}\right)$ of 1.6776 at the wavelength of $520 \mathrm{~nm}$, respectively. The strong optical anisotropy indicates that the host materials are horizontally oriented. 
The dipole orientation of the emitting dyes in the host matrix was determined from the analysis of the angle dependent PL spectrum of the EML using the classical dipole model. ${ }^{[18,}$ ${ }^{19]}$ The sample was prepared by co-depositing B3PYMPM, TCTA and $\operatorname{Ir}(\text { ppy })_{2}($ acac $)$ with the same molar ratio of the host materials and $8 \mathrm{wt} \%$ of the dopant on a pre-cleaned $1 \mathrm{~mm}$-thick fused silica substrate.The thickness of the film was $30 \mathrm{~nm}$, which corresponds to the thickness of the EML in the OLED. A He-Cd laser $(325 \mathrm{~nm})$ was used as the excitation source of the sample and the $p$-polarized emitted light at $520 \mathrm{~nm}$, corresponding to the peak wavelength of the PL spectrum of the phosphorescent dye, was detected. Details of the experimental method and the analysis are described in literature. ${ }^{[8]}$ In the simulation of the angle dependent PL spectrum, the optical anisotropy was accommodated in the model by using the effective refractive index as a function of the propagation direction of the $p$-polarized emitted light. The effective refractive index $\left(n_{\text {eff }}\right)$ is defined as a function of the emission angle $(\theta)$ in the organic layer as follows:

$$
\frac{1}{n_{e f f}^{2}}=\frac{\cos ^{2} \theta}{n_{o}^{2}}+\frac{\sin ^{2} \theta}{n_{e}^{2}}
$$

The effective refractive index of the organic layer for the $p$-polarized light changes from $n_{o}$ to $n_{e}$ as the angle of emission varies from $0^{\circ}$ to $90^{\circ}$. The experimental data and the simulation results of the angle dependent PL spectrum of the $30 \mathrm{~nm}$ thick $\operatorname{Ir}(\mathrm{ppy})_{2}(\mathrm{acac})$ doped TCTA:B3PYMPM film are shown in Fig. 1c. The experimental data are well fitted with the horizontal to vertical dipole ratio of 0.77:0.23, indicating that the emitter in the EML has more horizontally oriented dipoles than vertically oriented ones compared to the isotropic orientation and the result is consistent with the previous report. ${ }^{[12]}$ It is interesting to note that the $\Theta$ value is the same as that of $\operatorname{Ir}(\mathrm{MDQ})_{2}(\mathrm{acac}){ }^{[9-11]}$

\section{Results and discussion}


The device under investigation has a simple structure of glass/ITO $(70 \mathrm{~nm}) /$ TAPC (x nm)/TCTA (10 nm)/TCTA:B3PYMPM:Ir(ppy) 2 (acac) (1:1 of molar ratio and 8 wt.\%) (30 nm)/B3PYMPM (40 nm)/Al (100 nm), where TAPC represents 1,1-bis(di-4tolylaminophenyl)cyclohexane. The thickness of the TAPC layer was set as a parameter and varied from 40 to $100 \mathrm{~nm}$.

The current density-voltage-luminance $(J-V-L)$ characteristics of the devices with different thicknesses of the TAPC layer are shown in Fig. 2a. The $J-V$ characteristics of all the devices are not significantly different from each other, especially in the low current region, due to a high hole mobility of TAPC $\left(\sim 10^{-2} \mathrm{~cm}^{2} / \mathrm{Vs}\right) .{ }^{[26]}$ Thus, the difference in the efficiency of the OLEDs with different thicknesses of the TAPC layer originates mostly from an optical effect. The turn-on voltages of all the devices are identical at $2.4 \mathrm{~V}$ and the driving voltages of the devices are less than $3.9 \mathrm{~V}$ and $6.3 \mathrm{~V}$ for 1000 and $10000 \mathrm{~cd} / \mathrm{m}^{2}$, respectively. Maximum current efficiency and power efficiency are $106 \mathrm{~cd} / \mathrm{A}$ (60 nm-thick TAPC) and $127.3 \mathrm{~lm} / \mathrm{W}$ (80 nm-thick TAPC), respectively (not shown). The EQE of the devices corrected with their emission patterns (inset of Fig. 2b) are displayed in Fig. 2b. The maximum EQE of 30.2\% was obtained with the $80 \mathrm{~nm}$-thick TAPC layer. The EQE value is the highest one in bottom emitting green phosphorescent OLEDs based on an ITO electrode to our best knowledge. There is a paper reporting the EQE of $29.2 \%$ and $93 \mathrm{~cd} / \mathrm{A}$ using the same emitter. ${ }^{[27]}$ Unfortunately, the authors of that work did not take into account emitter orientation, which is obviously the key to achieve the high EQE. The measured EQE values of the devices are plotted in Fig. 2c against the thickness of the TAPC layer.

Optical simulation of the EQE of the devices was performed using the experimentally obtained values of $q_{P L}=0.94$ and $\Theta=0.77 \pm 0.02$. The PL quantum yield was measured using an integrating sphere ${ }^{[20,28]}$ and the sample consisted of the $50 \mathrm{~nm}$ thick emitting layer (TCTA:B3PYMPM:Ir(ppy) 2 (acac), 1:1 of molar ratio and 8 wt.\%) on a quartz substrate. The 
experimental results are very well described by the simulated results as shown in Fig. 2c under the condition of $\gamma=1$, indicating that the electrical loss is indeed negligible. In other words, the injected electrons and holes into the EML of the OLEDs efficiently recombine to form excitons. The excellent match between the experimental and the simulation results clearly indicates that the optical simulation describes the maximum achievable EQE under the known values of $q_{P L}$ and $\Theta$ when the device structure is optimized electrically and optically. This fact implies that we can predict the maximum EQE achievable with a certain emitting dye in a host by just measuring $q_{P L}$ and $\Theta$ on a neat film of the EML on glass without the need for fabrication of full OLED devices.

Based on this idea, we extend the simulation to calculate the maximum achievable EQEs as functions of $q_{P L}$ and $\Theta$. The corresponding simulation results are shown in Fig. 3 as a contour plot. We used the structure shown in Fig. 1a with the optimized thickness of the TAPC layer (75 nm) for the simulation. The maximum efficiency increases as $q_{P L}$ and $\Theta$ approach 1 as expected. Surprisingly, a maximum EQE of $46 \%$ can be achieved in normal ITO based bottom emitting OLEDs without any extra outcoupling layers using a phosphorescent dye with $q_{P L}=1$ and $\Theta=1$. Practically over $40 \% \mathrm{EQE}$ is possible with $q_{P L}=0.95$ and $\Theta=0.95$. In contrast, the maximum EQE of the OLED with isotropically oriented phosphorescent dyes is much lower $(\sim 25 \%)$.

\section{Conclusions}

In summary, optical analysis of the high efficiency OLED showed that the phosphorescent dye $\operatorname{Ir}(\mathrm{ppy})_{2}$ (acac) in the EML has a preferred horizontal dipole orientation (parallel to the substrate plane) to result in a maximum EQE of $30 \%$ under the condition of negligible electrical losses in the device. The prediction matches very well with the experimental value, 
suggesting that firstly the device has almost perfect electron-hole balance and the classical dipole model used for the calculation of $q_{\text {eff }}$ and $\eta_{\text {out }}$ are valid for the analysis of the performance of OLEDs. The analysis indicates that an EQE of $40 \%$ is possible in ITO based bottom emitting OLEDs without any extra light extraction layers.

\section{Experimental}

Device fabrication: The OLEDs were fabricated by thermal evaporation onto cleaned glass substrates pre-coated with ITO. Prior to the deposition of the organic layers, the ITO substrates were exposed to UV-ozone flux for 10 min following degreasing in acetone and isopropyl alcohol. All the layers were grown by thermal evaporation at a base pressure of $<$ $5 \times 10^{-7}$ Torr without breaking the vacuum.

Measurement of angle dependent PL intensity: The experimental setup was composed with a motorized rotation stage, a fused silica based half cylindrical lens with a holder of a sample, a dichroic mirror to filter the excitation beam, a polarizer to classify the polarity of emitted light, fiber guided detector combined with a monochromator and a photomultiplier tube. The continuous wave He-Cd laser (325 nm, Mellisgriot Co.) was used as the excitation source of the sample and their incident angle was 45 degree. The $p$-polarized emitted light at $520 \mathrm{~nm}$, corresponding to the peak wavelength of the PL spectrum of the phosphorescent dye, was detected.

Characterization of the OLEDs: The current density, the luminance, and the EL spectra were measured using a Keithley 2400 source meter and a SpectraScan PR650 (Photo Research). The angular distribution of the EL intensity was measured using the Keithley 2400 source meter, a rotation stage and an Ocean Optics S2000 fiber optic spectrometer. The EQE and the power efficiency of the OLEDs were calculated from the current density, the luminance, the EL spectra, and the angular distribution of the EL intensity. 
Measurement of PL quantum yield of the emitter: The PL efficiency of the emitter was measured using integrating sphere (Labsphere Co., 6 inches of diameter). He-Cd laser (325 $\mathrm{nm}$ ) was used as the excitation source, which is the same as the one used in the measurement of angle dependent PL intensity. A monochromator (Acton Research Co.) attached with a photomultiplier tube (Hamamatsu Photonics K.K.) was used as the optical detector system. To avoid the degradation of the samples by laser excitation, the samples were kept in inert environment by blowing nitrogen gas into the integrating sphere.

\section{Acknowledgements}

This work was supported by the industrial strategic technology development program [10035225, development of core technology for high performance AMOLED on plastic] funded by MKE/KEIT of Korea. C.M. acknowledges financial support by Deutscher Akademischer Austauschdienst (DAAD) and Bayerische Forschungsstiftung (BFS).

Received: ((will be filled in by the editorial staff))

Revised: ((will be filled in by the editorial staff)) Published online: ((will be filled in by the editorial staff))

[1] J.-S. Kim, P.K.H. Ho, N.C. Greenham and R.H. Friend, J. Appl. Phys., 2000, 88, 1073.

[2] P.K.H. Ho, J.-S. Kim, J.H. Burroughes, H. Becker, S.F.Y. Li, T.M. Brown, F. Cacialli and R.H. Friend, Nature, 2000, 404, 481.

[3] L.H. Smith, J.A.E. Wasey, I.D.W. Samuel and W.L. Barnes, Adv. Funct. Mater., 2005, $15,1839$.

[4] J.M. Ziebarth and M.D. McGehee, J. Appl. Phys., 2005, 97, 064502.

[5] M. Flämmich, M. C. Gather, N. Danz, D. Michaelis, A. H. Brauer, K. Meerholz and A. Tunnermann, Org. Electron., 2010, 11, 1039

[6] D. Yokoyama, A. Sakaguchi, M. Suzuki and C. Adachi, Org. Electron., 2009, 10, 127 
[7] D. Yokoyama, H. Sasabe, Y. Furukawa, C. Adachi and J. Kido, Adv. Funct. Mater., 2011, 21, 1375

[8] J. Frischeisen, D. Yokoyama, A. Endo, C. Adachi and W. Brütting, Org. Electron., 2011, 12,809

[9] M. Flämmich, J. Frischeisen, D. S. Setz, D. Michaelis, B. C. Krummacher, T. D. Schmidt, W. Brütting and N. Danz, Org. Electron., 2011, 12, 1663

10] T. D. Schmidt, D. S. Setz, M. Flämmich, J. Frischeisen, D. Michaelis, B. C. Krummacher, N. Danz and W. Brütting, Appl. Phys. Lett., 2011, 99, 163302

[11] L. Penninck, F. Steinbacher, R. Krause and K. Neyts, Org. Electron., 2012, 13 , 3079.

[12] P. Liehm, C. Murawski, M. Furno, B. Lüssem, K. Leo and M. C. Gather, Appl. Phys. Lett., 2012, 25, 253304

[13] W. Brütting, J. Frischeisen, T. D. Schmidt, B. J. Scholz and C. Mayr, Phys. Status Solidi A, 2012, 1 (2012)

[14] Y.-S. Park, S. Lee, K.-H. Kim, S.-Y. Kim, J.-H. Lee and J.-J. Kim, submitted

[15] T. Tsutsui, E. Aminaka, C. P. Lin and D. U. Kim, Philos. T. R. Soc. A, 1997, 355, 801

[16] M. Furno, R. Meerheim, S. Hofmann, B. Lüssem and K. Leo, Phys. Rev. B, 2012, 85, 115205

[17] E. M. Purcell, Phys. Rev., 1946, 69, 681

[18] R. R. Chance, A. Prock and R. Silbey, Molecular Fluorescence and Energy Transfer Near Interfaces. (John Wiley \& Sons, Inc., 1978)

[19] J. A. E. Wasey and W. L. Barnes, J. Mod. Opt., 2000, 47, 725

[20] W. I. Jeong, S. Y. Kim, J. W. Kang and J. J. Kim, Chem. Phys., 2009, 355, 25

[21] S. Y. Kim and J. J. Kim, Org. Electron., 2010, 11, 1010

[22] J. Lee, J. I. Lee, J. Y. Lee and H. Y. Chu, Org. Electron., 2009, 10, 1529 
[23] Refractive indices were measured by KRISS (Korea Research Institute of Standards and Science).

[24] E. D. Palik and G. Ghosh, Handbook of optical constants of solids. (Academic Press, San Diego, 1998).

[25] R. A. Synowicki, Thin Solid Films, 1998, 313, 394

[26] P. M. Borsenberger, L. Pautmeier, R. Richert and H. Bassler, J Chem Phys, 1991, 94, 8276.

[27] M. G. Helander, Z. B. Wang, J. Qiu, M. T. Greiner, D. P. Puzzo, Z. W. Liu and Z. H. Lu, Science, 2011, 332, 944.

[28] J.C. de Mello, H.F. Witmann and R.H. Friend, Adv. Mater., 1997, 9, 230. 
(a)
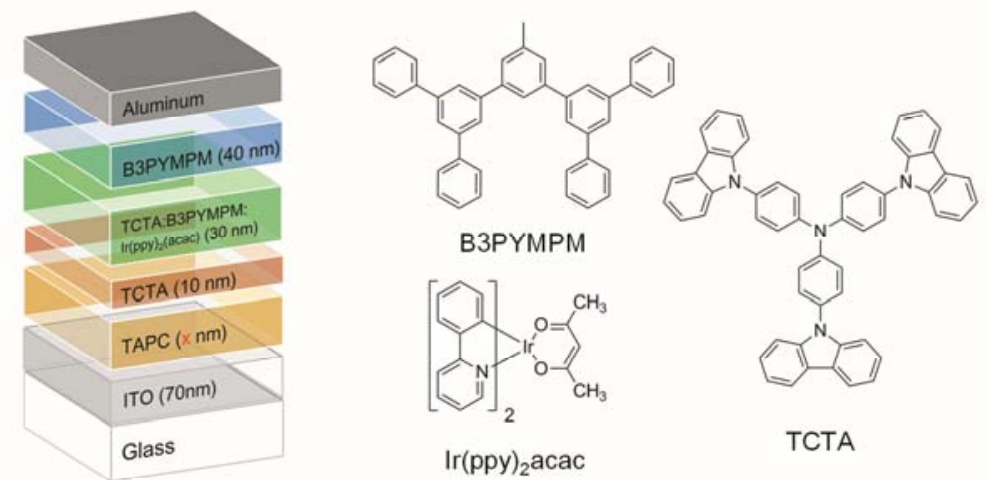

(b)

(c)
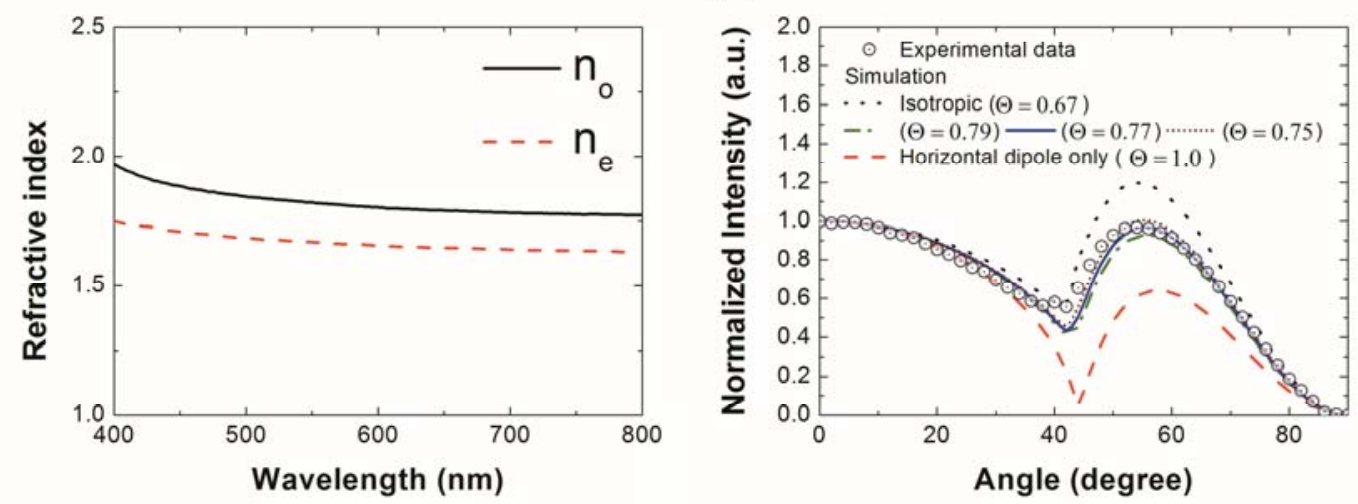

Figure 1. (a) Schematic diagram of the device and molecular structures of co-host and a phosphorescent dye used in this study. The thickness of the TAPC layer was varied from 40 to $100 \mathrm{~nm}$. (b) Refractive indices of undoped co-host layer (TCTA:B3PYMPM, 1:1 of molar ratio) measured by a variable angle spectroscopic ellipsometer. (c) Experimentally obtained angle dependent PL spectrum of EML on fused silica substrate (circle) is compared with the simulated ones (lines) with different portion of the horizontal dipoles $(\Theta)$ (red dashed line for $\Theta=1$ (fully horizontal), green dash-dot line for $\Theta=0.79$, blue thick-solid line for $\Theta=0.77$, purple short-dot line for $\Theta=0.75$, and black dotted line for $\Theta=0.67$ (isotropic)). 

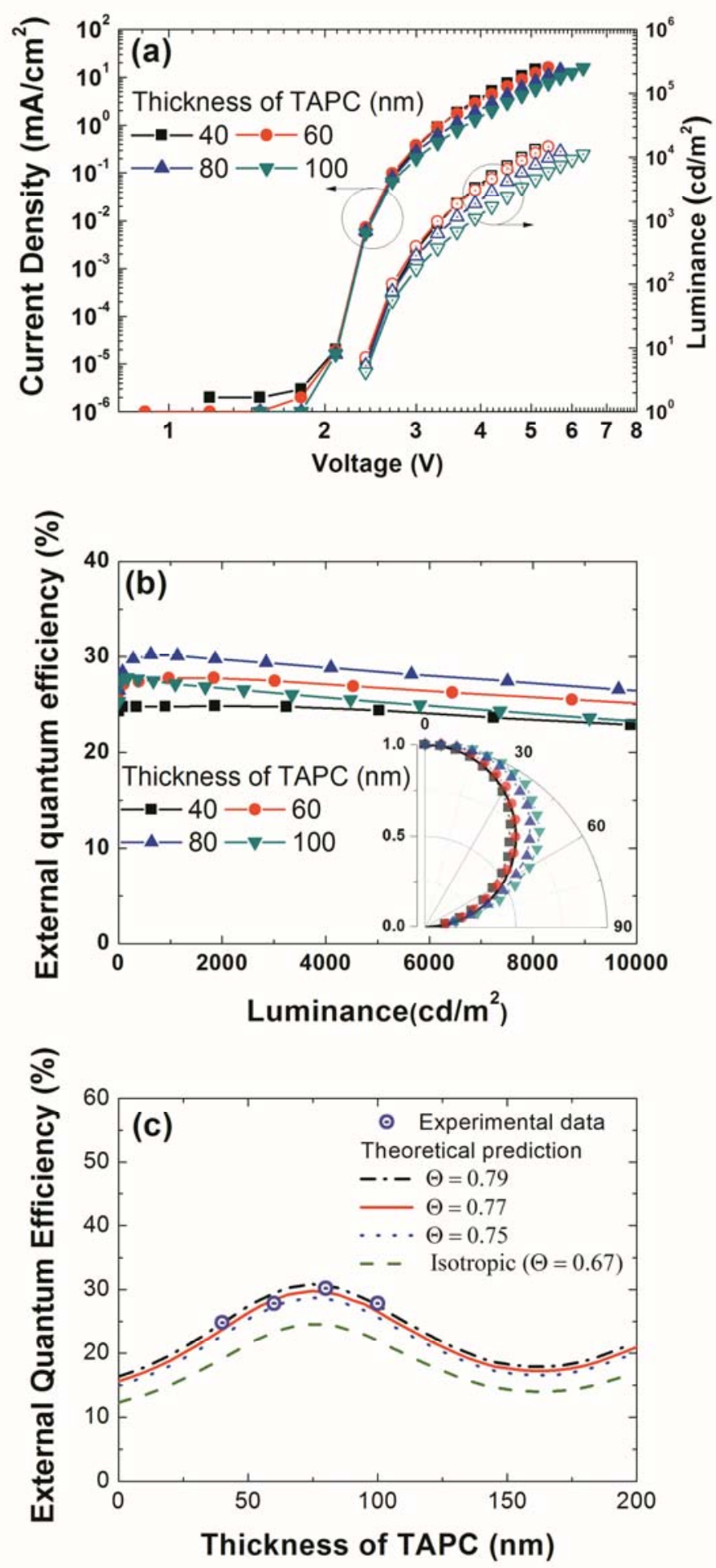

Figure 2. The experimental data of the OLEDs with different thicknesses of TAPC layer (40, 60, 80 and $100 \mathrm{~nm}$, respectively): (a) Current density-voltage-luminance curves (b) EQE vs. Luminance. (Inset: angle dependent emission patterns of the OLEDs. Black solid line indicates the Lambertian distribution). (c) Experimental EQEs (circles) are compared with simulated EQEs with different orientation factors of dipoles; black dash-dot line for $\Theta=0.79$, red solid line for $\Theta=0.77$, green dotted line for $\Theta=0.75$, and green dashed line for $\Theta=0.67$ (isotropic). $q_{P L}$ of 0.94 and $\gamma$ of 1.0 were used for the simulation. 


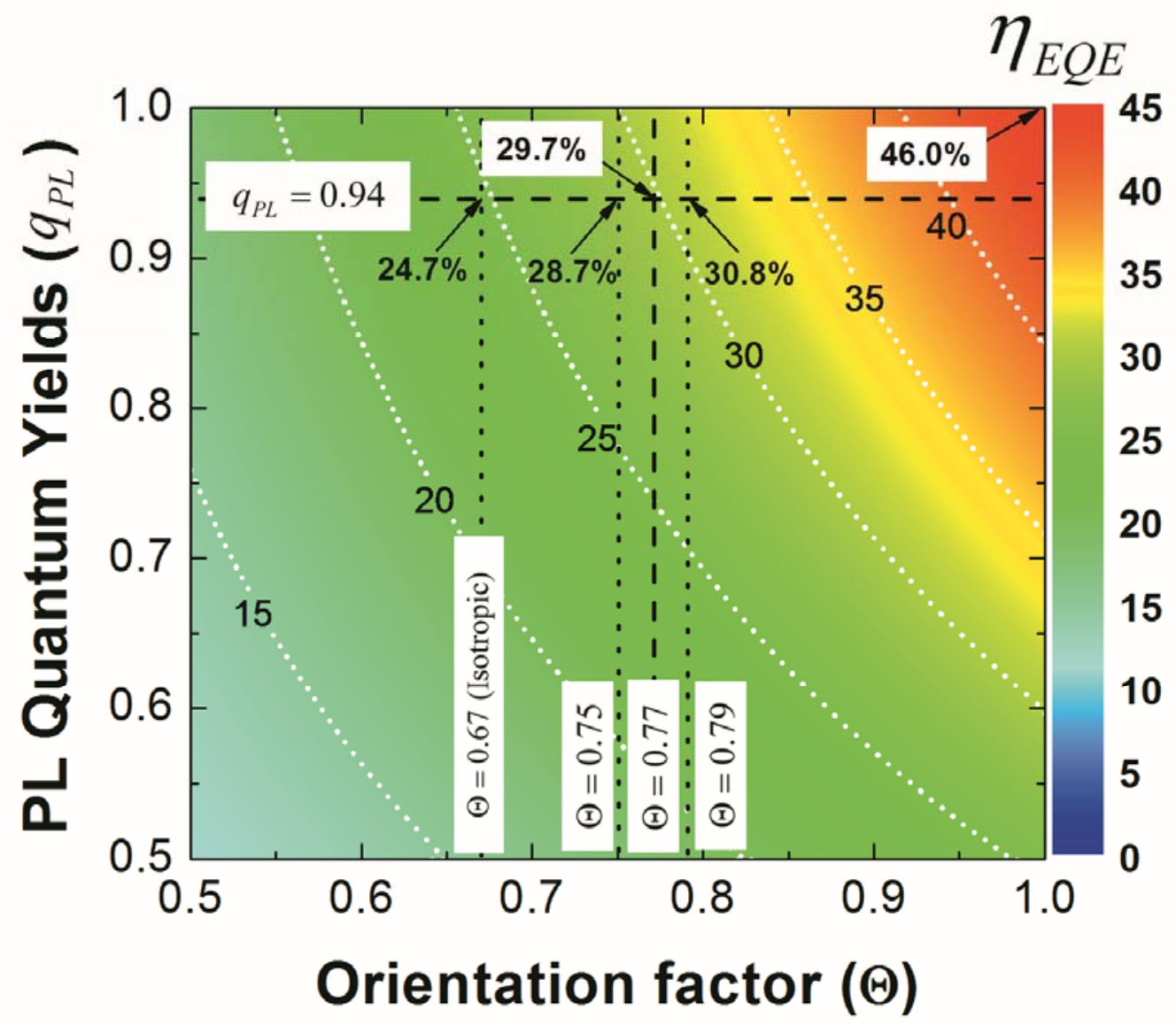

Figure 3. Contour plot of the simulation result of EQE as functions of $q_{P L}$ and $\Theta$. The device structure shown in Fig. 1 with TAPC thickness of $75 \mathrm{~nm}$ was used for the simulation. The dashed lines indicated the locus of EQE when $\Theta$ and $q_{P L}$ are 0.77 and 0.94 , respectively. In the same manner, the dotted lines indicate the EQE for $\Theta=0.67$ (isotropic), 0.75 and 0.79 , respectively. 


\section{The table of contents entry}

Using optical analysis we demonstrate that high efficiency phosphorescent OLEDs doped with $\operatorname{Ir}(\text { ppy })_{2}$ (acac) in an exciplex forming co-host have a preferred horizontal emitter orientation. Based on this analysis we calculate a maximum efficiency of the OLEDs of about $30 \%$ which matches very well with the experimental data. Furthermore, we suggest a simple method to predict the maximum efficiency achievable with a certain emitting dye in a host matrix.

Keyword: Organic light-emitting diodes, Phosphorescent emitter, Emitter orientation, Optical simulation, Ultra-high efficiency

Sei-Yong Kim, Won-Ik Jeong, Christian Mayr, Young-Seo Park, Kwon-Hyeon Kim, JeongHwan Lee, Chang-Ki Moon, Wolfgang Brütting, and Jang-Joo Kim*

\section{Title: Optical analysis of organic light-emitting diodes with $\mathbf{3 0 \%}$ external quantum efficiency}

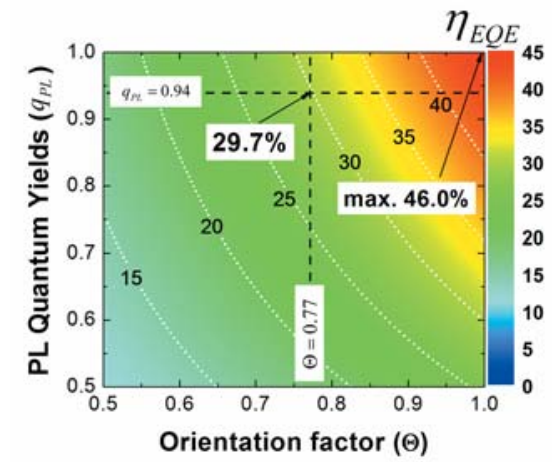

ToC figure ((Please choose one size: $55 \mathrm{~mm}$ broad $\times 50 \mathrm{~mm}$ high or $110 \mathrm{~mm}$ broad $\times 20 \mathrm{~mm}$ high. Please do not use any other dimensions)) 
Accepted for Adv. Funct. Mater. 23 (2013) 3896-3900

https://doi.org/10.1002/adfm.201300104

Supporting Information should be included here (for submission only; for publication, pleae provide Supporting Information as a separate PDF file). 